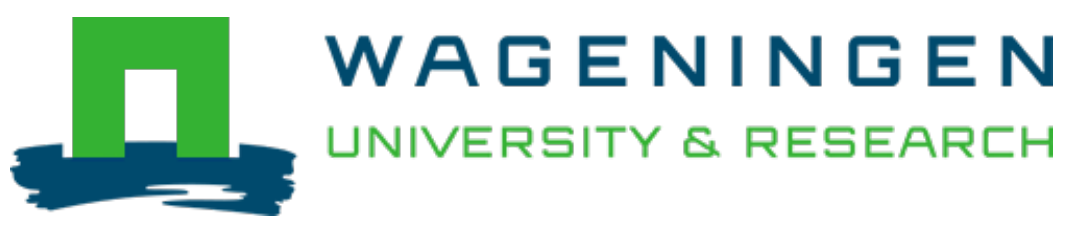

\title{
Interrelated Routes between the Maillard Reaction and Lipid Oxidation in Emulsion Systems
}

\author{
Journal of Agricultural and Food Chemistry \\ Troise, Antonio Dario; Fogliano, Vincenzo; Vitaglione, Paola; Berton-Carabin, Claire C. \\ https://doi.org/10.1021/acs.jafc.0c04738
}

This article is made publicly available in the institutional repository of Wageningen University and Research, under the terms of article $25 \mathrm{fa}$ of the Dutch Copyright Act, also known as the Amendment Taverne. This has been done with explicit consent by the author.

Article 25 fa states that the author of a short scientific work funded either wholly or partially by Dutch public funds is entitled to make that work publicly available for no consideration following a reasonable period of time after the work was first published, provided that clear reference is made to the source of the first publication of the work.

This publication is distributed under The Association of Universities in the Netherlands (VSNU) 'Article $25 \mathrm{fa}$ implementation' project. In this project research outputs of researchers employed by Dutch Universities that comply with the legal requirements of Article $25 \mathrm{fa}$ of the Dutch Copyright Act are distributed online and free of cost or other barriers in institutional repositories. Research outputs are distributed six months after their first online publication in the original published version and with proper attribution to the source of the original publication.

You are permitted to download and use the publication for personal purposes. All rights remain with the author(s) and / or copyright owner(s) of this work. Any use of the publication or parts of it other than authorised under article $25 \mathrm{fa}$ of the Dutch Copyright act is prohibited. Wageningen University \& Research and the author(s) of this publication shall not be held responsible or liable for any damages resulting from your (re)use of this publication.

For questions regarding the public availability of this article please contact openscience.library@,wur.nl 


\title{
Interrelated Routes between the Maillard Reaction and Lipid Oxidation in Emulsion Systems
}

\author{
Antonio Dario Troise,* Vincenzo Fogliano, Paola Vitaglione, and Claire C. Berton-Carabin
}

Cite This: J. Agric. Food Chem. 2020, 68, 12107-12115

Read Online

ABSTRACT: In foods, the Maillard reaction (MR) and lipid oxidation lead to the formation of several molecules through interrelated chemical pathways. MR and lipid oxidation products were investigated in model oil-in-water emulsions consisting of canola oil, water, and Tween 20, a nonionic surfactant, with glucose and phenylalanine. The presence of $1 \%$ Tween 20 , either in emulsion or as a control surfactant solution, sped up the formation of $N$-(1-deoxy-D-fructos-1-yl)-phenylalanine and of phenylacetaldehyde. Overall, the formation of MR products was up to sixteen times higher in emulsions than in an aqueous system without a surfactant. The formation of conjugated dienes, total aldehydes, hexanal, and $(Z)$-2-octenal was reduced down to six times when MR products were present in the emulsion. These results confirm that the formation of MR intermediates is influenced by the reactants' location, and the presence of a discrete nonpolar environment (oil droplets or surfactant micelles) promotes MR volatile formation through Strecker degradation.

KEYWORDS: Maillard reaction, lipid oxidation, emulsions, Amadori compounds, reactants' location

\section{INTRODUCTION}

Many foods contain lipids dispersed as droplets in an aqueous phase and are then oil-in-water $(\mathrm{O} / \mathrm{W})$ emulsions. The size of lipid droplets in these products typically ranges from $10^{-1}$ to $10^{2} \mu \mathrm{m} .{ }^{1}$ Especially, for small droplets, the developed surface area can reach great values (up to several $\mathrm{m}^{2}$ per gram oil), which tends to promote chemical reactions such as lipid oxidation, of which volatile end products may cause a loss of food quality. ${ }^{2}$ Factors such as the dimension of the lipid droplets, the type and concentration of emulsifiers, the possible formation of mixed lipid/surfactant micelles, the interactions between lipid droplets, and the chemical nature of antioxidants modulate the kinetics of the lipid oxidation reaction. ${ }^{3}$

The presence of protein is one of the most relevant factors to prevent lipid oxidation in emulsions, especially when present in large amounts that allow a substantial excess in the continuous phase, as they can efficiently scavenge free radicals and chelate transition metals. ${ }^{4}$ Many food-grade antioxidants are commercially available, but their efficacy remains systemdependent and thus difficult to generalize, as it is, at least in part, a consequence of the partition behavior between the polar and apolar phases of the systems. The relationship between chemical reactivity and reactant location is of pivotal importance in multiphase systems; therefore, characterizing the reactants' partitioning in the available phases is of utmost importance. ${ }^{5}$ Lipid droplets and surfactant micelles can segregate certain components (with moderate to high hydrophobicity) and possibly enhance their activity, by modulating the conventional reaction routes and defining new ones. ${ }^{6,7}$ Similarly, self-assembly of lipids in foods gives rise to novel structures that can promote sensory aspects of foods by increasing the release of aroma compounds. ${ }^{8}$ The interfacial layer plays a critical role in the definition of the interplay between lipid oxidation and the Maillard reaction (MR) in emulsions. Besides multiple evidence for the key role of the oil-water interface regarding lipid oxidation in emulsions, ${ }^{9}$ a gap of knowledge still exists on the interrelated mechanisms underpinning both reactions in dispersed systems and how the interface may be instrumental in that respect. Amphiphilic molecules with dual affinity for the lipid and aqueous regions can concentrate at the interface and enhance the reaction mechanisms for flavor formation, such as the Strecker degradation, or for antioxidant activity toward reactive hydroperoxides and carbonyls formed from lipid and sugar degradation, respectively. ${ }^{10,11}$

The concept of the so-called microreactors for Maillardderived flavors has already been described, and emulsions have been studied for their ability to improve the formation of volatile compounds. ${ }^{12}$ Focusing on the MR, applications range from the formation of sulfur-derived volatiles in structured fluids ${ }^{13}$ to the aroma composition in ghee (i.e., an inverse emulsion $)^{14}$ through the use of phospholipid- and monoglyceride-based self-assemblies for the formation of aroma compounds ${ }^{15}$ or to the formation of taste-active molecules as pyridinium betaines. ${ }^{16}$ The use of emulsions to control the MR was recently proposed looking at the Amadori compound formation as the starting point of the various reaction pathways: glycation rates were shown to depend on the presence of alternative environments of lower polarity than

Received: July 24, 2020

Revised: September 26, 2020

Accepted: October 2, 2020

Published: October 15, 2020

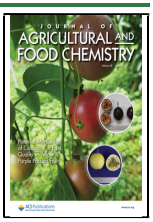


water, and the formation of MR intermediates may be envisioned as a starting point for protecting emulsions against deleterious oxidation reactions. ${ }^{17}$

In this paper, $\mathrm{O} / \mathrm{W}$ emulsions have been used as a tool to understand how the reactants' location controls the formation of Amadori compounds and Strecker aldehydes and how such intermediates and end products of the MR impact the oxidation of polyunsaturated fatty acids (PUFAs) present in the dispersed oil phase, through the measurement of conjugated dienes, lipid-derived aldehydes, hexanal, and $(Z)$ 2-octenal.

\section{MATERIALS AND METHODS}

Chemicals. Phenylalanine, ${ }^{13} \mathrm{C}$-phenylalanine, tyrosine, polyoxyethylene (20) sorbitan monolaurate (Tween 20), phenylacetaldehyde, 2-propanol, acetic acid, formic acid, and para-anisidine were obtained from Sigma-Aldrich (St. Louis, MO); D-glucose monohydrate and liquid chromatography mass spectrometry (LC-MS)-grade water were obtained from Merck (Darmstadt, Germany). Acetonitrile and $n$-hexane were obtained from Actu-All Chemicals (Oss, the Netherlands). Alumina powder was obtained from MP Biomedicals (Eschwege, Germany); N-(1-deoxy-D-fructos-1-yl)-L-phenylalanine (Fru-Phe) was obtained from Toronto Research Chemicals (Toronto, Canada). Canola oil was purchased from a local supermarket.

Preparation of Model Systems. Canola oil was stripped to remove endogenous antioxidants and surface-active compounds. Alumina powder was gently mixed with oil $(2: 1, \mathrm{w} / \mathrm{w})$ and stirred overnight at $100 \mathrm{rpm}$ in the dark to prevent oxidation and sedimentation. The alumina powder-oil mixture was then distributed in $50 \mathrm{~mL}$ conical tubes which were centrifuged at $2000 \mathrm{~g}$ for $20 \mathrm{~min}$. The supernatant was centrifuged a second time under the same conditions, after which the final clear supernatant, consisting of stripped canola oil, was transferred into a glass bottle and stored at $-20{ }^{\circ} \mathrm{C}$ until use. ${ }^{18}$ Three model systems (MSM) were prepared according to Table 1. MSM1 was made by solubilizing D-glucose

Table 1. Composition of the Five Model Systems Investigated. MSM4 and MSM5 Were Prepared to Assess (i) a Possible Antioxidant Effect of Phenylalanine toward Lipid Oxidation and (ii) the Intrinsic Effect of Tween 20 (and Tween 20 Micelles) on the Formation of Maillard Intermediates, Respectively $^{a}$

\begin{tabular}{ccccc}
$\begin{array}{c}\text { model } \\
\text { system }\end{array}$ & reactants & $\begin{array}{c}\text { lipid phase } \\
(10 \%)\end{array}$ & $\begin{array}{c}\text { surfactant } \\
(1 \%)\end{array}$ & \multicolumn{1}{c}{ target } \\
MSM1 & Phe/glucose & MR in aqueous \\
system \\
MSM2 & Phe/glucose & canola oil & Tween 20 & $\begin{array}{c}\text { MR and LO } \\
\text { interaction } \\
\text { LO in emulsified } \\
\text { system }\end{array}$ \\
MSM3 & canola oil & Tween 20 & $\begin{array}{c}\text { Phe oxidation in } \\
\text { emulsion }\end{array}$ \\
MSM4 & Phe & canola oil & Tween 20 & $\begin{array}{c}\text { MR in presence of } \\
\text { emulsifier }\end{array}$
\end{tabular}

${ }^{a}$ LO (lipid oxidation), MR (Maillard reaction), and Phe (phenylalanine).

monohydrate $(25 \mathrm{mM})$ and phenylalanine $(6.6 \mathrm{mM})$ in water. The mixture was stirred for $30 \mathrm{~min}$ at room temperature to fully dissolve both reagents. An aliquot of MSM1 was used to prepare MSM2 by incorporating $1 \%(\mathrm{w} / \mathrm{w})$ Tween 20 to ensure the stabilization of the large interfacial area and $10 \%(\mathrm{w} / \mathrm{w})$ stripped canola oil, while MSM3 was prepared with similar amounts of oil and surfactant but without the presence of glucose and phenylalanine in the aqueous phase. Two additional model systems, MSM4 and MSM5 analogous to MSM2 were also prepared. For MSM4, glucose was excluded to verify the ability of phenylalanine itself to exert any antioxidant activity in emulsions. For MSM5, stripped canola oil was not used, and the system was prepared by dissolving $1 \%$ Tween 20 in the aqueous phase with phenylalanine and glucose. Emulsification of MSM2, MSM3, and MSM4 was achieved in two steps. First, a coarse emulsion was obtained using a T-18 basic ULTRA-TURRAX high-shear mixer (IKA, Staufen im Breisgau, Germany) at 11,000 rpm for $40 \mathrm{~s}$. Then, the coarse emulsion was passed through a high-pressure homogenizer (Microfluidizer equipped with an F12Y interaction chamber; Microfluidics, Westwood, MA) at 800 bar, three times. The aqueous solutions MSM1 and MSM5 were also passed through the highpressure homogenizer under the same conditions to exclude any difference because of the high-pressure homogenization step.

Thermal Treatment and Incubation. An aliquot $(5 \mathrm{~mL})$ of each of the model systems was poured into a $9 \mathrm{~mL}$ screw-capped flask. Samples were heated in a heating block (Liebisch, Bielefeld, Germany) for $2,4,6$, and $8 \mathrm{~min}$ at $131{ }^{\circ} \mathrm{C}$ to allow the formation and degradation of Amadori compounds. After each time point of the thermal treatment, the heating block was equilibrated without samples for $5 \mathrm{~min}$ at $131{ }^{\circ} \mathrm{C}$. Samples were cooled down on ice immediately after heating and stored at $-20{ }^{\circ} \mathrm{C}$ until analyses. Thermally treated samples $\left(8 \mathrm{~min}\right.$ at $\left.131^{\circ} \mathrm{C}\right)$ were then incubated for $2,3,4,6$, and 7 days in a climate chamber (Memmert, Schwabach, Germany) at 40 ${ }^{\circ} \mathrm{C}$.

Physical Characterization of the Emulsion Systems. Particle Size and Particle Size Distribution. Physical characterization was performed on MSM2 and MSM3 after thermal treatment and over incubation. The mean particle diameter $\left(d_{3,2}\right)$, span, specific surface area, and particle size distribution of the emulsion systems were determined by static light scattering (Mastersizer 3000; Malvern Instruments, Malvern, U.K.). Values used for the refractive indices were 1.57 (red light) and 1.58 (blue light) for canola oil and 1.33 for water. The absorption index used was 0.01 (red and blue light). The samples were diluted in water prior to the measurement to obtain an obscuration between 5 and $7 \%$.

Microscopy Images. An Axio Scope.A1 microscope with a 100X objective lens and equipped with an Axiocam MRC5 (Zeiss, Jena, Germany) was used to obtain images of the emulsion systems. Emulsions were diluted 250 times in water, and one drop was placed on a glass slide and covered with a glass cover. Images were captured on three different locations of the emulsion systems.

Phenylalanine and Fru-Phe Quantitation. Quantitation of phenylalanine and Fru-Phe was achieved by liquid chromatography coupled to tandem mass spectrometry (LC-MS/MS), using ${ }^{13} \mathrm{C}$ phenylalanine as an internal standard. After thawing, the samples were centrifuged at $21,700 \mathrm{~g}$ for $10 \mathrm{~min}$ and the aqueous phase was filtered using modified cellulose syringe filters (RC, $0.2 \mu \mathrm{m}$, Phenomenex, Torrance, CA) and finally diluted 150 times in water. The separation of phenylalanine and Fru-Phe was performed using an Ultimate 3000 UHPLC (Thermo Fisher, Bremen, Germany) coupled to a TSQ Quantum ultra tandem mass spectrometer (Thermo Fisher). A Kinetex C18 EVO column $(100 \mathrm{~mm} \times 2.1 \mathrm{~mm}, 2.6 \mu \mathrm{m}$, Phenomenex) was used. The mobile phases consisted of $0.1 \%$ formic acid in water (A) and $0.1 \%$ formic acid in acetonitrile (B) with the following gradient flow $(\% \mathrm{~B} / \mathrm{min})$ : $(2 / 0),(2 / 3.8),(65 / 5.5)$, and $(65 / 7.5)$ at $0.2 \mathrm{~mL} / \mathrm{min}$. Five microliters of the sample was injected and the column temperature was set at $20{ }^{\circ} \mathrm{C}$. Positive heated electrospray ionization was used for detection with the following source parameters: spray voltage $3.2 \mathrm{kV}$; capillary temperature 350 ${ }^{\circ} \mathrm{C}$; vaporizer temperature $250{ }^{\circ} \mathrm{C}$; scan time $0.2 \mathrm{~s}$; and sheath gas and auxiliary gas 35 and 30 arbitrary units, respectively. Chromatograms were recorded in the selected reaction monitoring (SRM) mode, and the characteristic transitions and their respective collision energy (CE) were monitored to improve selectivity: phenylalanine $(\mathrm{m} / z 166$ $\rightarrow$ 120, 103; CE: 29, 13), ${ }^{13} \mathrm{C}$-phenylalanine $(m / z 167 \rightarrow 103,120$; CE: 13, 28), and Fru-Phe $(m / z 328 \rightarrow 292$, 310; CE: 8, 15), in bold, the transitions used for qualification. Within the same chromatographic run, an additional SRM experiment was inserted to monitor the oxidation of phenylalanine into tyrosine using the following transition: $(m / z 182 \rightarrow 165$; CE: 25$)$. Fru-Phe, phenylalanine, and tyrosine calibration curves were built in ranges $50-10,000 \mathrm{ng} / \mathrm{mL}$ and 
Table 2. Measured Physical Properties of the O/W Emulsions during the Thermal Treatment $\left(131{ }^{\circ} \mathrm{C}\right)$ and Subsequent Incubation $\left(40{ }^{\circ} \mathrm{C}\right)^{a}$

\begin{tabular}{|c|c|c|c|c|c|}
\hline MSM & time & $d_{(4,3)}(\mu \mathrm{m})$ & $d_{(3,2)}(\mu \mathrm{m})$ & span & $A\left(\mathrm{~m}^{2} / \mathrm{g}\right)$ \\
\hline \multicolumn{6}{|c|}{ Thermal Treatment, $130{ }^{\circ} \mathrm{C}$} \\
\hline MSM2 & $0 \min$ & $0.26( \pm 0.01)$ & $0.17( \pm 0.01)$ & $1.66( \pm 0.07)$ & $38.9( \pm 0.4)$ \\
\hline MSM2 & 2 & $0.82( \pm 0.50)$ & $0.22( \pm 0.05)$ & $4.54( \pm 2.87)$ & $31.4( \pm 7.4)$ \\
\hline MSM2 & 4 & $1.07( \pm 0.57)$ & $0.34( \pm 0.15)$ & $2.26( \pm 0.02)$ & $23.6( \pm 10.2)$ \\
\hline MSM2 & 6 & $1.39( \pm 0.90)$ & $0.47( \pm 0.28)$ & $3.81( \pm 1.96)$ & $21.0( \pm 12.5)$ \\
\hline MSM2 & $8 \mathrm{~min}$ & $1.85( \pm 0.61)$ & $0.61( \pm 0.28)$ & $5.18( \pm 5.62)$ & $16.4( \pm 8.3)$ \\
\hline MSM3 & $0 \mathrm{~min}$ & $0.25( \pm 0.01)$ & $0.17( \pm 0.01)$ & $1.58( \pm 0.05)$ & $38.3( \pm 1.97)$ \\
\hline MSM3 & 2 & $0.76( \pm 0.01)$ & $0.21( \pm 0.01)$ & $10.76( \pm 0.06)$ & $31.8( \pm 0.04)$ \\
\hline MSM3 & 4 & $0.77( \pm 0.36)$ & $0.22( \pm 0.03)$ & $6.91( \pm 5.56)$ & $30.7( \pm 3.97)$ \\
\hline MSM3 & 6 & $1.46( \pm 1.06)$ & $0.28( \pm 0.09)$ & $6.56( \pm 5.63)$ & $26.4( \pm 8.21)$ \\
\hline MSM3 & $8 \mathrm{~min}$ & $1.99( \pm 0.75)$ & $0.42( \pm 0.19)$ & $7.28( \pm 5.15)$ & $19.0( \pm 8.02)$ \\
\hline \multicolumn{6}{|c|}{ Incubation, $40{ }^{\circ} \mathrm{C}$} \\
\hline MSM2 & 0 day & $1.85( \pm 0.61)$ & $0.61( \pm 0.28)$ & $5.81( \pm 5.62)$ & $16.4( \pm 8.9)$ \\
\hline MSM2 & 1 & $1.46( \pm 0.75)$ & $0.61( \pm 0.40)$ & $5.55( \pm 3.83)$ & $18.1( \pm 11.9)$ \\
\hline MSM2 & 2 & $1.30( \pm 0.55)$ & $0.54( \pm 0.33)$ & $5.84( \pm 3.40)$ & $18.4( \pm 11.1)$ \\
\hline MSM2 & 3 & $1.85( \pm 0.12)$ & $0.99( \pm 0.41)$ & $1.68( \pm 0.28)$ & $17.9( \pm 3.3)$ \\
\hline MSM2 & 6 & $1.83( \pm 0.76)$ & $0.63( \pm 0.29)$ & $4.69( \pm 4.04)$ & $14.9( \pm 10.0)$ \\
\hline MSM2 & 7 days & $10.00( \pm 8.70)$ & $0.65( \pm 0.06)$ & $4.41( \pm 2.38)$ & $10.4( \pm 1.0)$ \\
\hline MSM3 & 0 day & $1.99( \pm 0.75)$ & $0.42( \pm 0.19)$ & $7.28( \pm 5.15)$ & $19.0( \pm 8.0)$ \\
\hline MSM3 & 1 & $1.22( \pm 1.18)$ & $0.52( \pm 0.32)$ & $3.36( \pm 1.12)$ & $20.3( \pm 7.7)$ \\
\hline MSM3 & 2 & $1.56( \pm 1.05)$ & $0.30( \pm 0.11)$ & $3.51( \pm 1.80)$ & $24.7( \pm 8.7)$ \\
\hline MSM3 & 3 & $3.27( \pm 0.64)$ & $0.60( \pm 0.15)$ & $2.82( \pm 0.65)$ & $11.6( \pm 2.5)$ \\
\hline MSM3 & 6 & $1.68( \pm 0.79)$ & $0.44( \pm 0.22)$ & $6.39( \pm 4.36)$ & $19.2( \pm 8.4)$ \\
\hline MSM3 & 7 days & $11.55( \pm 9.80)$ & $0.62( \pm 0.21)$ & $4.43( \pm 1.30)$ & $11.9( \pm 3.3)$ \\
\hline
\end{tabular}

${ }^{a}$ The specific surface area $A$, or interfacial area per mass unit of the dispersed phase $\left(\mathrm{m}^{2} / \mathrm{g}\right)$ was calculated according to the following equation: $A=$ $3 / r \times \rho$ ( $r$, radius; $\rho$, density). MSM stands for model system; for their respective compositions, see Table 1 . For thermal treatment, time refers to min, while for incubation, it refers to days.

$10-500 \mathrm{ng} / \mathrm{mL}$, respectively, according to the limit of detection and limit of quantification (LOQ). Three replicates of the $0.5 \mathrm{ng} / \mathrm{mL}$ solutions were injected into the ultraperformance liquid chromatography-tandem mass spectrometer system to verify the lowest concentration for which the signal-to-noise ratio was $>3$. Concentrations of $<0.1 \mathrm{ng} / \mathrm{mL}$ resulted in no signal and the LOQ was $5 \mathrm{ng} /$ $\mathrm{mL}$ for the standard solution of phenylalanine and Fru-Phe. Reproducibility of the method was evaluated through an intraday and an interday assay. The slope among the three subsequent calibration curves had an RSD below $7 \%$.

Determination of Volatile Compounds. Solid-phase microextraction coupled to gas chromatography and mass spectrometry (SPME-GC-MS) was used to quantify the phenylacetaldehyde produced by the Strecker degradation of phenylalanine, as well as hexanal and (Z)-2-octenal. A TRACE 1300 GC (Thermo Fisher) coupled to an ISQ single quadrupole MS system (Thermo Fisher) and to an RXI-5 ms $(20 \mathrm{~m} \times 0.18 \mathrm{~mm}$ internal diameter, film thickness $0.36 \mu \mathrm{m}$, Restek, Bellefonte, PA) was used. Volatile compounds extraction was achieved using a polydimethylsiloxane fiber (coating thickness $100 \mu \mathrm{m}$, Sigma-Aldrich). Samples were heated at $60{ }^{\circ} \mathrm{C}$ in a Peltier heater with a preincubation time of $2 \mathrm{~min}$ and a speed of $350 \mathrm{rpm}$. The extraction time was $10 \mathrm{~min}$ followed by a desorption time of $29 \mathrm{~min}$. Cryogenics was used to focus the volatiles and the injection was in the splitless mode with a splitless time of 2.5 min and a split flow of $25 \mathrm{~mL} / \mathrm{min}$. The purge flow was $5.0 \mathrm{~mL} / \mathrm{min}$ with constant septum purge. Oven temperature started at $40{ }^{\circ} \mathrm{C}$ and was held for $4 \mathrm{~min}$ and then it increased with $10{ }^{\circ} \mathrm{C} / \mathrm{min}$ to $250{ }^{\circ} \mathrm{C}$ and this temperature was held for $5 \mathrm{~min}$. The ion source temperature and the MS transfer line temperature were both $250{ }^{\circ} \mathrm{C}$. Helium was used as a carrier gas with a constant flow rate of $0.8 \mathrm{~mL} / \mathrm{min}$. The ionization mode was EI $(70 \mathrm{eV})$ in the full scan mode $(33-300 \mathrm{~m} / z)$ with a scan time of $0.2 \mathrm{~s}$. A calibration curve was built to quantify volatiles in the range of $0.1-10 \mathrm{mg} / \mathrm{kg}$. For MSM1, the calibration curve was prepared in water, and for model systems with stripped canola oil, the calibration curve was prepared in a blank emulsion. The coefficient of correlation was higher than 0.98 for both calibration curves. Compounds were identified based on their mass spectra using AMDIS (National Institute of Standards and Technology, Gaithersburg, MD). Additional control for the identification of the volatile compounds was performed by injection of the alkane series from $\mathrm{C} 1$ to $\mathrm{C} 20$.

Determination of Conjugated Dienes. Conjugated dienes were measured in MSM2, MSM3, and MSM4 in triplicate as primary markers of PUFA oxidation. An aliquot of $50 \mu \mathrm{L}$ of the emulsion was collected in a tube to which $950 \mu \mathrm{L}$ of 2-propanol was added. After vigorous mixing, samples were further diluted 20 times in 2-propanol and then centrifuged for $4 \mathrm{~min}$ at $1200 \mathrm{~g}$. The supernatant was collected in quartz cuvettes (Hellma Analytics, Müllheim Germany) and the absorbance spectra were measured in the range $200-310 \mathrm{~nm}$ using a DU 720 UV/VIS spectrophotometer (Beckman Coulter, Brea, $\mathrm{CA}$ ). The absorbance at $233 \mathrm{~nm}$ was used to determine the concentration of conjugated dienes using a molar extinction coefficient of $27,000 \mathrm{M}^{-1} \mathrm{~cm}^{-1}$ at $233 \mathrm{~nm}$. The concentration was reported in $\mathrm{mmol} / \mathrm{kg}$ of oil.

Determination of Total Aldehydes. The para-anisidine value (pAV) was measured in duplicate to determine the formation of total aldehydes, which are secondary lipid oxidation products (LOPs). An aliquot of $0.6 \mathrm{~g}$ of the emulsion was weighed in a $5 \mathrm{~mL}$ tube and $3 \mathrm{~mL}$ of the $n$-hexane/2-propanol mixture $(3: 1, \mathrm{v} / \mathrm{v})$ was added to the emulsion. The tube was then vortexed to induce phase separation, with the oil solubilized in the clear upper hexane phase. The absorbance of $1 \mathrm{~mL}$ of this upper hexane layer $(\mathrm{Ab})$ was measured at $350 \mathrm{~nm}$ using hexane as a blank. One milliliter of the hexane phase was collected and $0.2 \mathrm{~mL}$ of para-anisidine solution $(2.5 \mathrm{~g} / \mathrm{L}$ in acetic acid) was added to each tube. After vortexing and waiting for $10 \mathrm{~min}$, the absorbance (As) was measured at $350 \mathrm{~nm}$ using a blank prepared by mixing $1 \mathrm{~mL}$ of pure hexane with $0.2 \mathrm{~mL}$ of para-anisidine solution. The pAV (arbitrary units) was then calculated as follows (eq 1) 

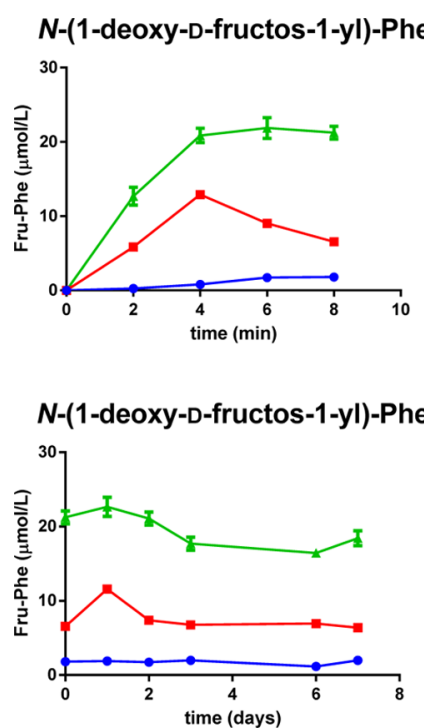

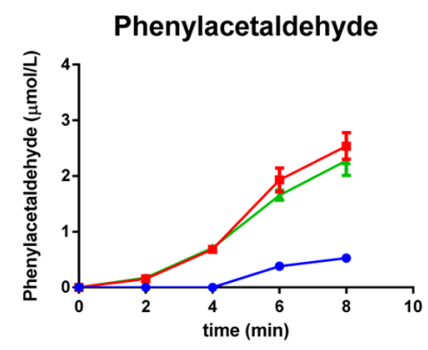

Phenylacetaldehyde

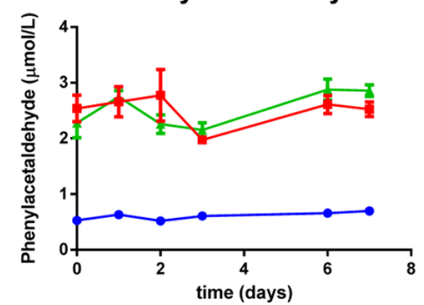

Phenylalanine

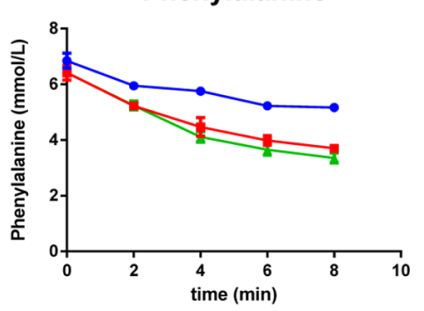

Phenylalanine

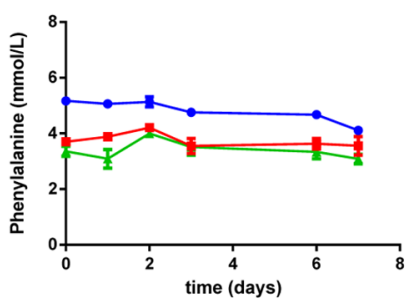

Figure 1. Amadori compound (N-(1-deoxy-D-fructos-1-yl)-phenylalanine, Fru-Phe) and Strecker aldehyde (phenylacetaldehyde) formation and phenylalanine degradation during thermal treatment at $131{ }^{\circ} \mathrm{C}$ for up to $8 \mathrm{~min}$ (upper panel) and during incubation at $40{ }^{\circ} \mathrm{C}$ for up to 7 days (bottom panel), in emulsion (MSM2, red squares), water (MSM1, blue circles), and nonionic surfactant solution (MSM5, green triangles). Significant differences (Tukey test, $\alpha<0.05$ ) between the target compounds in the three model systems are listed in Table S1.

$$
\mathrm{pAV}=\frac{(1.2 \times \mathrm{As}-\mathrm{Ab})}{m}
$$

where $m$ is the mass of oil per $\mathrm{mL}$ of hexane $(\mathrm{g} / \mathrm{mL})$.

Statistical Analysis. Target analytes, pAV, conjugated dienes, physicochemical parameters, phenylalanine and its Amadori compound, phenylacetaldehyde, hexanal, and $(Z)$-2-octenal were monitored in four independently prepared $\mathrm{O} / \mathrm{W}$ emulsions and control systems upon incubation and thermal treatment. The results were expressed as $\mathrm{mM}$ for phenylalanine or $\mu \mathrm{M}$ for Fru-Phe, phenylacetaldehyde andtyrosine, while the other two volatile compounds were reported in $\mathrm{ppm}$. The time/course profile and the Tukey test for statistical analysis $(\alpha=0.05)$ were monitored using GraphPad (Prism, La Jolla, CA).

\section{RESULTS AND DISCUSSION}

Physicochemical Attributes of Emulsified Systems. Table 2 shows the influence of thermal treatment at $131{ }^{\circ} \mathrm{C}$ and subsequent incubation at $40{ }^{\circ} \mathrm{C}$ for 7 days on the physicochemical characteristics of both emulsion model systems. Particle size distribution, Sauter mean diameter, span, and surface area were measured over time. In both MSM2 and MSM3 (emulsions with and without Maillard precursors, respectively), the droplet size increased upon thermal treatment and this effect was more pronounced in MSM2 (emulsions with phenylalanine and glucose). In this system, the specific surface area accordingly decreased during the thermal treatment, from $38.9 \mathrm{~m}^{2} / \mathrm{g}$ oil down to $16.4 \mathrm{~m}^{2} / \mathrm{g}$ oil after $8 \mathrm{~min}$, while in MSM3, the specific surface area was reduced from $38.3 \mathrm{~m}^{2} / \mathrm{g}$ oil down to $19.0 \mathrm{~m}^{2} / \mathrm{g}$. The influence of thermal treatment on the droplet size was confirmed by the increase in span, an index of the polydispersity of emulsions. When emulsions were subsequently incubated at $40{ }^{\circ} \mathrm{C}$, for the first 6 days, no significant changes in the droplet size and related parameters were observed (Table 2). At day 7 , in both MSM2 and MSM3, we measured a value of $\left(d_{4,3}\right)$ higher than $10 \mu \mathrm{m}$ and a decrease in the specific surface area of around $37-38 \%$ compared to day 0 . Although it may be questionable whether Tween 20 micelles still exist as such during the heat treatment, when checking emulsion morphology by light microscopy, no droplets with a radius larger than $10 \mu \mathrm{m}$ were visible in the unheated emulsions, while when MSM2 and MSM3 were heated for $8 \mathrm{~min}$ at $131{ }^{\circ} \mathrm{C}$, a few larger droplets became visible (Figure S1). The microscopic appearance of emulsions did not change during subsequent incubation at 40 ${ }^{\circ} \mathrm{C}$. Microscopy images excluded that flocculation was responsible for the observed increase in the droplet size upon heat treatment; indeed, the large spherical droplets that appeared rather point to at coalescence. Coalescence in emulsions subjected to a high-temperature treatment can be explained looking at the cloud point of Tween 20 and at the radius of droplets. A partial dehydration of the hydrophilic polyoxyethylene chains at temperatures close to the cloud point of the surfactant $\left(79.6{ }^{\circ} \mathrm{C}\right)$ generates a marked decrease in the hydrophilicity of the polar portion resulting in a reduced surface coverage of the emulsion droplets and in an increase in interfacial tension. ${ }^{19-21}$ Moreover, it cannot be excluded that Tween 20-degradation products, such as hydroperoxides and short chain fatty acids, affected physiochemical attributes. ${ }^{22}$ The physical destabilization of emulsion droplets after thermal treatment and incubation is in line with results obtained from model emulsions containing Tween $20^{23}$ and coconut milk emulsions. ${ }^{24}$ MSM2 and MSM3 were characterized by an initial droplet radius lower than $100 \mathrm{~nm}$, probably resulting in collisions between droplets with high frequencies as a consequence of the large Brownian motion. ${ }^{25}$

Formation of MR Products. To investigate how the MR was influenced by a multiphase environment, the formation and elimination of the Amadori product (Fru-Phe) and the formation of phenylalanine-derived Strecker aldehyde (phenylacetaldehyde) were monitored. Their concentrations during the thermal treatment at $131{ }^{\circ} \mathrm{C}$ are outlined in Figure 1 (top panels). The presence of Tween 20 micelles in MSM5 clearly accelerated the formation of Fru-Phe: the concentration of the Amadori compound was higher than $20 \mu \mathrm{M}$ at $4 \mathrm{~min}$ and it remained at least twice higher than in MSM2 up to the end of the thermal treatment. In the emulsion system with glucose and phenylalanine (MSM2), Fru-Phe formation was around five and four times higher than in MSM1 at 6 and $8 \mathrm{~min}$, 

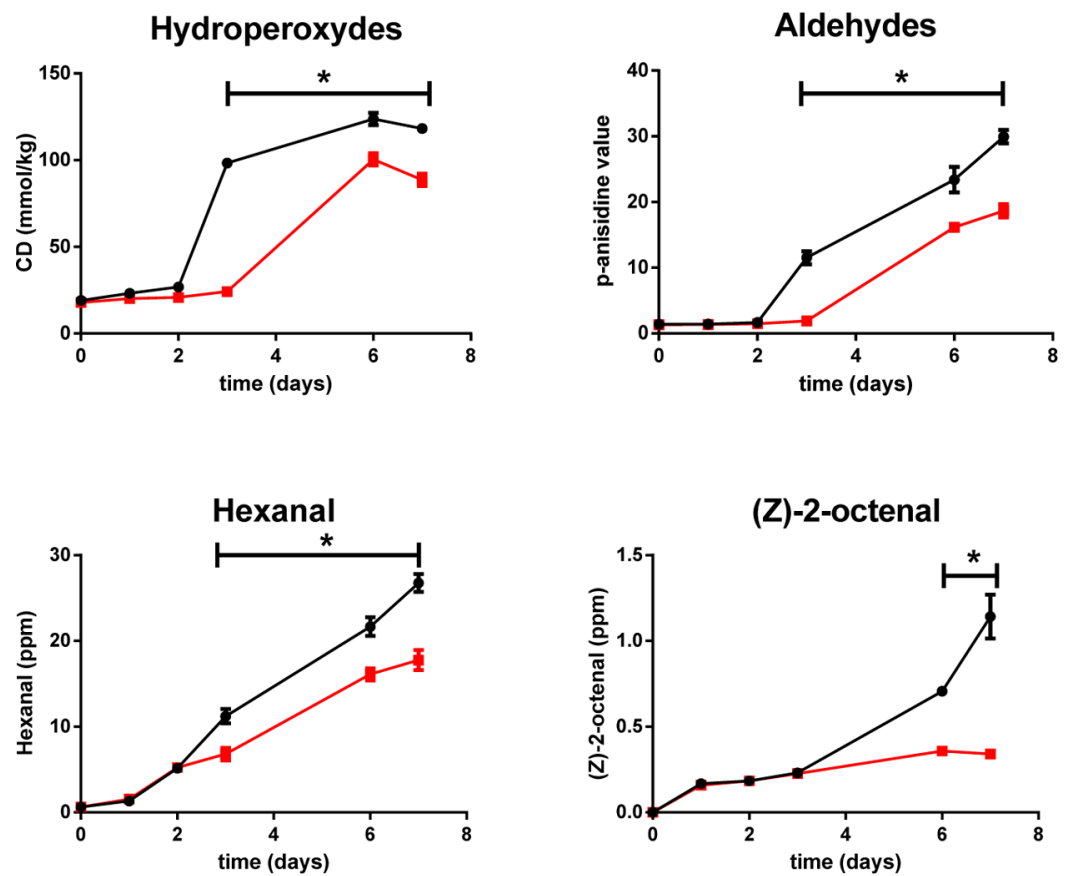

(Z)-2-octenal

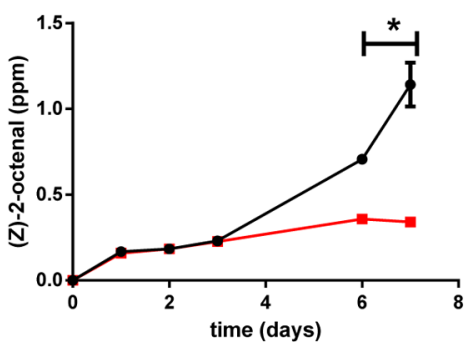

Figure 2. Markers of primary lipid oxidation (conjugated dienes) and secondary lipid oxidation (para-anisidine value; hexanal and 2-octenal) during 7 days incubation at $40{ }^{\circ} \mathrm{C}$ of emulsions containing MR products (MSM2, red squares) or of emulsions without Maillard precursors and intermediates (MSM3, black circles). Asterisks $(*)$ indicate a significant difference (Tukey test, $\alpha<0.05$ ) between the target compounds in both emulsion systems.

respectively. The highest concentration was $12.908 \pm 0.135$ $\mu \mathrm{M}$ at $4 \mathrm{~min}$, after which degradation of this adduct prevailed on formation. Fru-Phe slightly increased during thermal treatment in the water model system (MSM1) and its concentration at $8 \mathrm{~min}$ was $1.729 \pm 0.024 \mu \mathrm{M}$. Phenylacetaldehyde concentration substantially increased in all three tested systems, and as for the Amadori compound, the presence of Tween 20 and/or of emulsion droplets promoted the Strecker degradation as compared to the water model system (MSM1). Already after $4 \mathrm{~min}$ at $131{ }^{\circ} \mathrm{C}$, phenylacetaldehyde concentrations in MSM1 and MSM2 were significantly different, while at $8 \mathrm{~min}$, phenylacetaldehyde was five times higher in the emulsion system (MSM2) than in the water control (MSM1). In MSM5, phenylacetaldehyde concentration was in line with that in MSM2, suggesting that the favored formation of Strecker aldehydes was due to the nonionic surfactant for a large part. It should be pointed out that the chosen Tween 20 concentration $(1 \% \mathrm{w} / \mathrm{w})$ is by orders of magnitude higher than its critical micelle concentration ( $\mathrm{cmc}$; around 0.05 to $0.08 \mathrm{mM}$ at room temperature, according to Kim and Hsieh). ${ }^{26}$ This means that Tween 20 micelles were overwhelmingly present in both MSM5 and in the aqueous phase of MSM2. At $8 \mathrm{~min}$ at 131 ${ }^{\circ} \mathrm{C}$, phenylalanine concentration was reduced by 24 and $38 \%$ of its initial value in aqueous and emulsion systems, respectively, while in the presence of Tween 20 without oil (MSM5), phenylalanine concentration was reduced by $40 \%$ compared to MSM1. These three markers thus clearly and consistently indicate that the presence of a nonionic surfactant concentration largely above the $\mathrm{cmc}$ greatly promotes the glycation reaction during a high-temperature thermal treatment, both in a micellar solution and in a surfactant-stabilized emulsion.

During incubation at $40{ }^{\circ} \mathrm{C}$ (Figure 1, bottom panels), in MSM2 and MSM5, Fru-Phe increased within the first hours of incubation reaching the maximum concentration after the first day, followed by a decrease down to a plateau value around 6 $\mu \mathrm{M}$ until day 7. In MSM5, the concentration was around three times higher than in MSM2. Phenylacetaldehyde exhibited a slight increase at the beginning of the incubation and then decreased, whereas no significant change over time was recorded in MSM1. The phenylacetaldehyde concentration in the emulsion system (MSM2) remained significantly higher than in the water model system (MSM1, see also Table S1).

During the incubation, the differences in the concentration of phenylalanine in MSM2 and MSM5 were significant only at day 1 and at day 7, while in MSM1, the difference with the two model systems containing Tween 20 (MSM2 and MSM5) remained significant throughout the incubation at $40{ }^{\circ} \mathrm{C}$. From a mechanistic point of view, phenylalanine decay in MSM5, MSM1, and MSM2 was in line with previous findings: the presence of glucose favored the formation of the glycosylamine and its rearrangement into the Amadori compound. ${ }^{27}$ Fru-Phe formation in the aqueous system followed the same trends previously observed: first, the formation of the Amadori product and then its degradation over time. ${ }^{17}$ In this previous work using a comparable procedure, we also investigated the reactivity of phenylalanine and glucose in emulsions with tricaprylin as the oil phase (i.e., saturated medium-chain triglycerides, which therefore are not subjected to lipid oxidation). Interestingly, both phenylalanine and Fru-Phe in the presence of tricaprylin showed a different behavior toward canola oil: differences in Fru-Phe concentration with tricaprylin were significant only at $4 \mathrm{~min}$, while Fru-Phe concentrations at the other time points and the profile of phenylalanine decrease in watery medium and emulsions were the same. The physical properties of canola oil and tricaprylin and the formation of carbonyl compounds during thermal treatment can be responsible for different reaction kinetics and the consequent higher formation of the Amadori compound. 


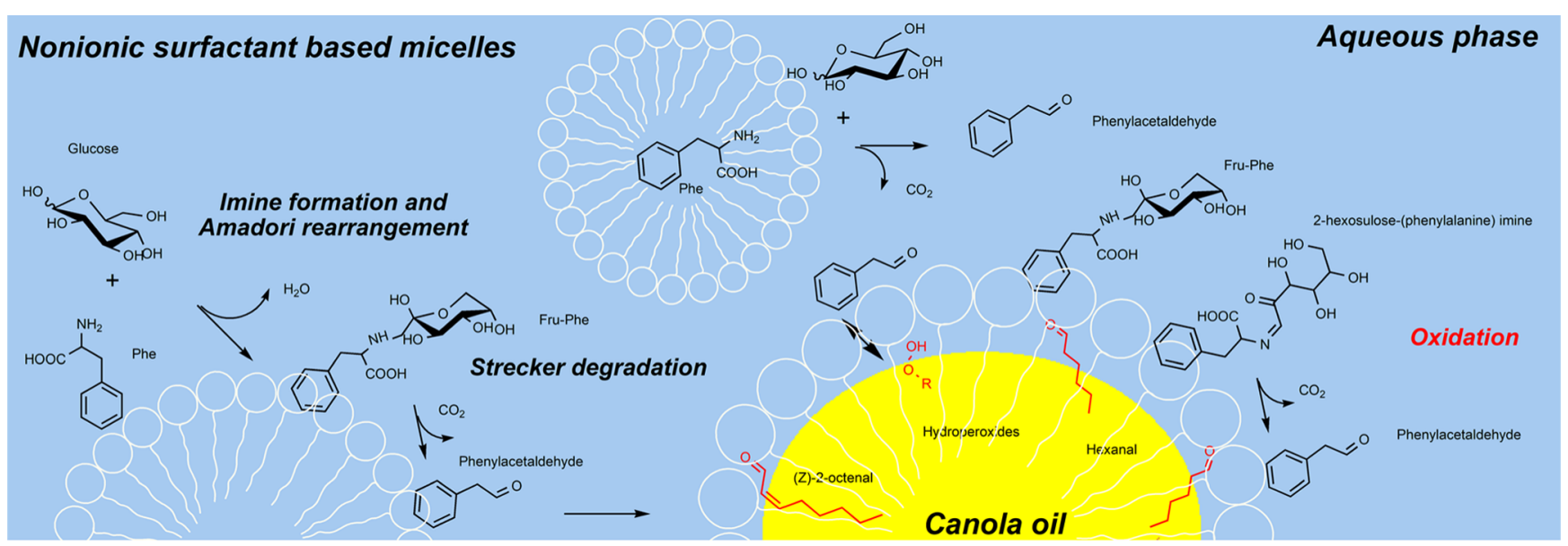

Figure 3. Proposed location and mechanisms for Maillard precursors, intermediate, and end products and their interplay with the formation of LOPs. Phenylalanine is located at the oil-water interface, upon reaction with glucose, the Amadori compound (Fru-Phe) is formed via imine formation and then oxidized into Strecker aldehyde (phenylacetaldehyde) via 2-hexosulose-(phenylalanine) imine formation. In red, LOPs (a generic hydroperoxide, hexanal, and $(Z)$-2-octenal) are schematized along with their possible interactions with Maillard products at the interface.

These results confirm the potential of submicron emulsion droplets and micelles to modulate the MR. The amphiphilic nature of phenylalanine made a key difference in comparison with the use of precursors with very $\operatorname{low} \log P$, in theory located in the aqueous phase: the location at the interface with an anisotropic orientation toward the aqueous environment resulted in a five fold increase in phenylacetaldehyde in the presence of Tween 20, with or without canola oil droplets. The higher formation of phenylacetaldehyde in emulsified system than the aqueous system followed similar results of flavor formation in the presence of monoglyceride and phospholipidbased self-assembly: a 1.7- and 3-fold increase in norfuraneol concentration was observed in reverse hexagonal compared with the aqueous phase, using xylose/leucine and xylose/ glycine, respectively. ${ }^{28}$ Concerning the formation of phenylacetaldehyde, Fru-Phe was the key intermediate. ${ }^{29}$ We hypothesize that the presence of lipids promoted the oxidative degradation of Fru-Phe following a mechanism similar to what was reported for oxygen and transition metals: Fru-Phe can be oxidized into the 2-hexosulose-(phenylalanine) imine as a consequence of LOPs' formation, which represents the key step responsible for the favored formation of phenylacetaldehyde from the Amadori compound. ${ }^{30}$

Next to Strecker degradation and Amadori compound formation, we also considered the oxidation of phenylalanine as a possible route for aromatic amino acid decrease, as oxidation may occur also on the aromatic ring of phenylalanine, leading to the formation of tyrosine. ${ }^{31}$ In this respect, we prepared a new model system MSM4 with phenylalanine and emulsified canola oil. Tyrosine was included in SRM experiments and no significant changes were observed during thermal treatments in both emulsion-based system and aqueous environment, while an amount of tyrosine close to $0.25 \mu \mathrm{M}$ was already formed at time 0 , suggesting that oxidation during the homogenization step cannot be excluded, while during thermal treatment, glycation and Strecker degradation prevailed on direct conversion of phenylalanine into tyrosine (Figure S2). This finding supports the hypothesis that the most relevant pathway of phenylalanine degradation was the reaction with the reducing carbonyl of glucose. Figure S2 reports the concentration of tyrosine in MSM4: no significant changes were observed, and the aromatic amino acids were almost constant during thermal treatment and incubation with a slight decrease after the last 2 days of incubation.

Formation of LOPs. Lipid oxidation was investigated by measuring conjugated dienes, which are primary LOPs and secondary LOPs: total aldehydes via the pAV and volatile compounds (hexanal and (Z)-2-octenal) in emulsions preliminary subjected to $8 \mathrm{~min}$ of thermal treatment at 131 ${ }^{\circ} \mathrm{C}$, subsequently incubated at $40{ }^{\circ} \mathrm{C}$ (Figure 2). The amount of primary LOPs increased during incubation at $40{ }^{\circ} \mathrm{C}$ in both emulsions yet to a lower extent in MSM2 (containing MR precursors and products) than in MSM3 (not containing phenylalanine and glucose). At day 3, this difference was particularly clear as the amount of primary LOPs formed was four times higher in MSM3 compared to MSM2. The results for the pAV were in line with the results for conjugated dienes. Until 3 days, no increase in the formation of total aldehydes was observed in MSM2, while the pAV in MSM3 was around six times higher than MSM2. The formation of volatile LOPs was also in line with the results of conjugated dienes and pAV. On the first 3 days, no difference between both model systems was observed; however, after 3 days, the concentrations of hexanal and ( $Z$ )-2-octenal in MSM3 were twice higher than those in MSM2. It is worth mentioning that the preliminary heat treatment did not show a direct effect on the amount of primary LOPs and significant differences between MSM2 and MSM3 were visible only after the induction phase. The amount of conjugated dienes changed from $13.55 \pm 0.80$ to $13.92 \pm 1.57 \mathrm{mmol} / \mathrm{kg}$ for MSM2 and from $12.47 \pm 1.44$ to $14.43 \pm 1.06 \mathrm{mmol} / \mathrm{kg}$ for MSM3, highlighting no significant increase. The formation of secondary LOPs during thermal treatment was less relevant than during incubation: the pAV increased from $0.325 \pm 0.026$ to $1.538 \pm 0.075$ for MSM2 and from $0.348 \pm 0.037$ to $0.953 \pm 0.072$, even including aldehydes formed in the MR pathways and extracted in the hexane phase. Besides the slight variations observed, the heat treatment up to 8 min may form a small amount of lipid radicals which could next rapidly enter the propagation phase, thereby promoting lipid oxidation during the subsequent incubation at $40{ }^{\circ} \mathrm{C}$. To investigate if phenylalanine alone was able to prevent lipid oxidation to a significant extent, additional control experiments were included. Along with the measurement of tyrosine 
(Figure S2), conjugated dienes were measured at the end of incubation in an emulsion containing phenylalanine but not glucose, hence, without the formation of Fru-Phe and advanced end products: in that system (MSM4), the final concentration of LOPs was significantly higher than those in the model systems MSM3 and MS2, respectively (Figure S3). This indicates that under the experimental conditions used, in emulsions, phenylalanine itself did not exhibit any substantial antioxidant effect.

Therefore, the formation of aromatic amino acid-carbonyl degradation products significantly reduced the concentration of all the markers of lipid oxidation. This effect was recently highlighted for $\mathrm{O} / \mathrm{W}$ emulsions stabilized by whey proteindextran: the MR interfacial layer promoted an effective steric hindrance to the initial oxidation stage through antioxidant steric hindrance, and in the intermediate stage of the oxidation process, the adsorption at the interface of the emulsion prevented free radical chain reactions while the unabsorbed Maillard end products protected oil from oxidation. ${ }^{32}$ Dong and co-workers demonstrated that caramelization and the MR both contributed to the increase in antioxidant activity in fish $\mathrm{O} / \mathrm{W}$ emulsions stabilized with $1 \%$ Tween 20 , but hydrolyzed $\beta$-lactoglobulin and glucose Maillard end products were able to effectively reduce lipid hydroperoxides upon a thermal treatment up to $2 \mathrm{~h}$ at $90{ }^{\circ} \mathrm{C}$; longer thermal treatment was as effective as $\beta$-lactoglobulin without sugar. ${ }^{33}$

Interplay between the MR and Lipid Oxidation. Figure 3 depicts how the reactant location may boost reaction mechanisms in both Maillard and lipid oxidation pathways in $\mathrm{O} / \mathrm{W}$ emulsions. We propose that a local accumulation and molecular orientation of phenylalanine at the surface of emulsion droplets would be a relevant factor promoting the interaction of the amino group with glucose, leading to the increased formation of the Amadori compound. In MSM2 and MSM5, the micellar structure of the Tween 20 fraction presumably promoted the synthesis of Fru-Phe through the location of phenylalanine at the interface of the micelle where it can be attached by the carbonyl moiety of glucose, promoting the formation of the Amadori compound and of its degradation/oxidation products, such as phenylacetaldehyde. A comparable behavior was already outlined by Baglioni and co-workers, ${ }^{34}$ suggesting that the hydrophobic region of phenylalanine can be positioned in between the head groups of the nonionic surfactant. The efficiencies of carbonyl groups in reacting with the free amino groups may depend not only on the rates of the chemical reactions involved (as observed in MSM1), but also on the amino group concentrations in the different regions of the emulsion and how the relative polarities of the oil, nonionic surfactant, and water affected the rate of Amadori rearrangement. The faster conversion in MSM5 can be explained also with the continuous exchange of phenylalanine during collision of the aggregates Tween 20/phenylalanine. The increase in the Amadori compound concentration during the thermal treatment can suggest some similarities with the antioxidant mechanisms in lipid dispersions and location at the interface. ${ }^{5,35}$ In parallel, hydrophilic Amadori compounds are formed at higher yields when amino acid precursors are located at the interface; indeed, phenylalanine underwent faster carbonyl attachment in the presence of emulsion or surfactants droplets. This may be understood based on its partition coefficient $(\log P: 0.235 \pm 0.277$, SciFinder, CAS) resulting in an anchoring to the Tween 20 micelles' surface and orientation to the aqueous phase, making it prone to carbonyl attachment. A similar behavior was proposed by Garti and co-workers for cysteine and furfural in an $\mathrm{O} / \mathrm{W}$ emulsion system with nonionic surfactants. ${ }^{36}$ The remarkable enhancement of the formation rate and the distribution of different flavor products in $\mathrm{O} / \mathrm{W}$ media were related to the compartmentalization of the reactants at the interface parallel to the reaction carried out in the aqueous phase. This effect accelerated bimolecular reactions by acting to concentrate locally the reactants and providing a large contact between reducing carbonyls and the amino group of phenylalanine.

The propensity of nonionic surfactants in modulating chemical reactions was already demonstrated for lipid oxidation. For instance, the antioxidant activity of tocopherols was enhanced by adding Tween 20 to $\mathrm{O} / \mathrm{W}$ emulsions. ${ }^{37}$ The addition of excess nonionic surfactants to the aqueous phase of emulsions and the ability of surfactants to form micelles may slow down lipid oxidation through chelation of metal ions, scavenging of free radicals, and/or segregation of lipid oxidation primary products from the oil droplets. ${ }^{9}$ Conversely, in model systems MSM2 and MSM5, a Tween 20-based interface most likely favored the accumulation and improved the solubilization and stabilization of Maillard intermediates such as Fru-Phe and phenylacetaldehyde, as occur for MR taste-active molecules in a eutectic solvent. ${ }^{38}$ Both Fru-Phe and phenylacetaldehyde decreased reaction rates of hydroperoxide and aldehyde formation in MSM2. The enhanced reaction rates in MSM2 and MSM5 are consequently in line with the pseudophase kinetic model: association colloids in a homogeneous aqueous solution are conceptually modeled as separate phases within the aqueous phase. ${ }^{39}$ It is possible to also speculate on the location and reactivity of phenylalanine in Tween 20-stabilized emulsions: a combination of both a lipid phase and a Tween 20-based interface provides a microenvironment able to accumulate an aromatic amino acid according to its partition coefficient, leading to high concentrations of Fru-Phe and phenylacetaldehyde. A similar behavior was demonstrated regarding the accumulation of antioxidants in the reaction region. ${ }^{40}$

The relationship between Maillard intermediates and end products in emulsions provided a new perspective for the control of lipid oxidation. Besides the well-known antioxidant activity of MR products and melanoidins, in particular, ${ }^{41,42}$ low-molecular-weight compounds impaired the formation of LOPs via metal-chelating capacity and radical-scavenging ability. ${ }^{43}$ The formation of amine-carbonyl condensation products explained why Fru-Phe and its end products were more effective than the precursor. In particular, Osada and Shibamoto demonstrated that volatile extracts from the MR model system were able to totally block hexanal development, ${ }^{44}$ while Chen and Kitts indicated that high-molecularweight Maillard products did not necessarily show higher antioxidant activity compared to low-molecular-weight compounds. ${ }^{45}$ Other hypotheses encompass the possible interaction of Fru-Phe and other small MR degradation products with lipid-derived carbonyls. The release of lipid-derived aldehydes and carbonyls mainly occurs at the interface, ${ }^{9}$ where the concentration of MR products can be higher, thus resulting in the reduction of lipid oxidation. The interfacial location of Maillard-derived antioxidants promotes oxidative stability as they were located near the initiation reaction site and these results confirmed that both reactions predominantly took place at the interface according to the partitioning of 
precursors. ${ }^{46}$ Analogous to the partitioning of amino acids, the esterification of hydroxycinnamic acid derivatives offers a smart example regarding the association between antioxidant activity and location in emulsions: an alkyl chain with 8-12 carbon atoms exhibits an effective protection against lipid oxidation, while longer or shorter chains are partitioned into lipid and aqueous layer, respectively, resulting in a decay of the reaction at the interface. ${ }^{5}$ Interrelated routes between the MR and lipid oxidation and the compounds formed can be part of the colloidal ecosystem typical of $\mathrm{O} / \mathrm{W}$ emulsions: in colloidal dispersions, the dynamic trafficking of surfactants, lipid droplets, and water-soluble reactive molecules can be notably influenced by micelle protection, polarity, and partitioning changes in the MR intermediates as recently reported for LOPs. $^{47}$

In this paper, we demonstrated that emulsions and nonionic surfactant micelles were able to promote the carbonyl-amine reaction resulting in higher concentration of Fru-Phe and Strecker aldehyde compared to a simple water phase, which can have direct consequences on the formation of the aroma active compounds. The local accumulation of antioxidant Maillard-derived compounds exhibited an effective protection toward the formation of LOPs, including volatiles typically associated with off-flavors such as hexanal and (Z)-2-octenal. We propose that compartmentalization and partitioning of reactive species in the presence of a discrete nonpolar phase can be used to regulate the formation of desired and undesired compounds. A dual benefit can be achieved in systems where MR intermediates promote the formation of desirable volatiles and prevent lipid oxidation.

\section{ASSOCIATED CONTENT}

\section{SI Supporting Information}

The Supporting Information is available free of charge at https://pubs.acs.org/doi/10.1021/acs.jafc.0c04738.

Optical microscopy images of emulsions; concentration of tyrosine in the control aqueous model system, the emulsion with both MR precursors, and the emulsion with phenylalanine and without glucose; concentration of conjugated dienes in the emulsion with both phenylalanine and glucose, the control emulsion with no MR precursors, and the emulsion with phenylalanine and without glucose at the end of incubation at $40{ }^{\circ} \mathrm{C}$; and two-way ANOVA (PDF)

\section{AUTHOR INFORMATION}

\section{Corresponding Author}

Antonio Dario Troise - Proteomics \& Mass Spectrometry Laboratory, ISPAAM, National Research Council, 80055 Naples, Italy; Department of Agricultural Sciences, University of Naples Federico II, 80055 Naples, Italy; $\odot$ orcid.org/00000001-7635-5244; Phone: +39 064993 27732;

Email: antoniodario.troise2@unina.it

\footnotetext{
Authors

Vincenzo Fogliano - Food Quality and Design Group, Wageningen University, 6708 WG Wageningen, The Netherlands; o orcid.org/0000-0001-8786-9355

Paola Vitaglione - Department of Agricultural Sciences, University of Naples Federico II, 80055 Naples, Italy
}

Claire C. Berton-Carabin - Food Process Engineering Group, Wageningen University, 6708 WG Wageningen, The Netherlands; INRAE, UR BIA, F-44316 Nantes, France

Complete contact information is available at: https://pubs.acs.org/10.1021/acs.jafc.0c04738

\section{Funding}

The research of Antonio Dario Troise was supported by University of Naples (UNINA, Italy) and Compagnia di San Paolo, in the frame of Program "STAR-L1" (Sostegno Territoriale alle Attività di Ricerca, Junior principal investigator grants, University of Naples, Italy).

\section{Notes}

The authors declare no competing financial interest.

\section{ACKNOWLEDGMENTS}

We thank Max Wijkamp and Raquel Valentín Martínez at Food Quality and Design group and Food Process Engineering group, Wageningen University, for their valuable help with the model system preparation and GC-MS experiments.

\section{ABBREVIATIONS}

MR, Maillard reaction; Fru-Phe, N-(1-deoxy-D-fructos-1-yl)-Lphenylalanine; MS/MS, tandem mass spectrometry; LOPs, lipid oxidation products; pAV, para-anisidine value

\section{REFERENCES}

(1) Coupland, J. N.; McClements, D. J. Lipid Oxidation in Food Emulsions. Trends Food Sci. Technol. 1996, 7, 83-91.

(2) Waraho, T.; Mcclements, D. J.; Decker, E. A. Mechanisms of Lipid Oxidation in Food Dispersions. Trends Food Sci. Technol. 2011, 22, 3-13.

(3) Decker, E. A.; McClements, D. J.; Bourlieu-Lacanal, C.; Durand, E.; Figueroa-Espinoza, M. C.; Lecomte, J.; Villeneuve, P. Hurdles in Predicting Antioxidant Efficacy in Oil-in-Water Emulsions. Trends Food Sci. Technol. 2017, 67, 183-194.

(4) Faraji, H.; McClements, D. J.; Decker, E. A. Role of Continuous Phase Protein on the Oxidative Stability of Fish Oil-in-Water Emulsions. J. Agric. Food Chem. 2004, 52, 4558-4564.

(5) Laguerre, M.; Bayrasy, C.; Panya, A.; Weiss, J.; McClements, D. J.; Lecomte, J.; Decker, E. A.; Villeneuve, P. What Makes Good Antioxidants in Lipid-Based Systems? The Next Theories Beyond the Polar Paradox. Crit. Rev. Food Sci. Nutr. 2015, 55, 183-201.

(6) Capuano, E.; Oliviero, T.; van Boekel, M. A. J. S. Modeling Food Matrix Effects on Chemical Reactivity: Challenges and Perspectives. Crit. Rev. Food Sci. Nutr. 2017, 58, 2814-2828.

(7) Zhao, Y.; Elias, R. J.; Coupland, J. N. Effect of Food Structure on the Distribution and Reactivity of Small Molecules. Curr. Opin. Food Sci. 2015, 4, 19-24.

(8) Sagalowicz, L.; Michel, M.; Blank, I.; Schafer, O.; Leser, M. E. Self-Assembly in Food - A Concept for Structure Formation Inspired by Nature. Curr. Opin. Colloid Interface Sci. 2017, 28, 87-95.

(9) Berton-Carabin, C. C.; Ropers, M.-H.; Genot, C. Lipid Oxidation in Oil-in-Water Emulsions: Involvement of the Interfacial Layer. Compr. Rev. Food Sci. Food Saf. 2014, 13, 945-977.

(10) Mezzenga, R.; Seddon, J. M.; Drummond, C. J.; Boyd, B. J.; Schröder-Turk, G. E.; Sagalowicz, L. Amphiphilic Lipids: NatureInspired Design and Application of Lipidic Lyotropic Liquid Crystals. Adv. Mater. 2019, 31 (). DOI: 10.1002/adma.201970250.

(11) Hidalgo, F. J.; Zamora, R. Interplay between the Maillard Reaction and Lipid Peroxidation in Biochemical Systems. Ann. N.Y. Acad. Sci. 2005, 1043, 319-326.

(12) Garti, N. Microemulsions as Microreactors for Food Applications. Curr. Opin. Colloid Interface Sci. 2003, 8, 197-211. 
(13) Vauthey, S.; Milo, C.; Frossard, P.; Garti, N.; Leser, M. E.; Watzke, H. J. Structured Fluids as Microreactors for Flavor Formation by the Maillard Reaction. J. Agric. Food Chem. 2000, 48, 4808-4816.

(14) Newton, A. E.; Fairbanks, A. J.; Golding, M.; Andrewes, P.; Gerrard, J. A. The Influence of Emulsion Structure on the Maillard Reaction of Ghee. Food Chem. 2015, 173, 1243-1249.

(15) Sagalowicz, L.; Moccand, C.; Davidek, T.; Ghanbari, R.; Martiel, I.; Negrini, R.; Mezzenga, R.; Leser, M. E.; Blank, I.; Michel, M. Lipid Self-Assembled Structures for Reactivity Control in Food. Philos. Trans. R. Soc., A 2016, 374, 20150136.

(16) Troise, A. D.; Berton-Carabin, C. C.; Vitaglione, P.; Fogliano, V. Formation of Taste-Active Pyridinium Betaine Derivatives Is Promoted in Thermally Treated O/W Emulsions and Alkaline $\mathrm{pH}$. J. Agric. Food Chem. 2020, 68, 5180-5188.

(17) Troise, A. D.; Berton-Carabin, C. C.; Fogliano, V. Amadori Products Formation in Emulsified Systems. Food Chem. 2016, 199, 51-58.

(18) Berton, C.; Genot, C.; Ropers, M.-H. Quantification of Unadsorbed Protein and Surfactant Emulsifiers in Oil-in-Water Emulsions. J. Colloid Interface Sci. 2011, 354, 739-748.

(19) Chawla, J.; Mahajan, R. K. Cloud Point Studies of Tween and Glycol in the Presence of Salts. J. Dispersion Sci. Technol. 2011, 32, $822-827$.

(20) Davis, H. T. Factors Determining Emulsion Type: HydrophileLipophile Balance and Beyond. Colloids Surf., A 1994, 91, 9-24.

(21) Shinoda, K.; Carlsson, A.; Lindman, B. On the Importance of Hydroxyl Groups in the Polar Head-Group of Nonionic Surfactants and Membrane Lipids. Adv. Colloid Interface Sci. 1996, 64, 253-271.

(22) Kerwin, B. A. Polysorbates 20 and 80 Used in the Formulation of Protein Biotherapeutics: Structure and Degradation Pathways. J. Pharm. Sci. 2008, 97, 2924-2935.

(23) Demetriades, K.; McClements, D. J. Influence of PH and Heating on Physicochemical Properties of Whey Protein-Stabilized Emulsions Containing a Nonionic Surfactant. J. Agric. Food Chem. 1998, 46, 3936-3942.

(24) Tangsuphoom, N.; Coupland, J. N. Effect of Thermal Treatments on the Properties of Coconut Milk Emulsions Prepared with Surface-Active Stabilizers. Food Hydrocolloids 2009, 23, 17921800.

(25) Raikos, V. Effect of Heat Treatment on Milk Protein Functionality at Emulsion Interfaces. A Review. Food Hydrocolloids 2010, 24, 259-265.

(26) Kim, C.; Hsieh, Y.-L. Wetting and Absorbency of Nonionic Surfactant Solutions on Cotton Fabrics. Colloids Surf., A 2001, 187$188,385-397$.

(27) Ge, S.-J.; Lee, T.-C. Kinetic Significance of the Schiff Base Reversion in the Early-Stage Maillard Reaction of a PhenylalanineGlucose Aqueous Model System. J. Agric. Food Chem. 1997, 45, $1619-1623$.

(28) Blank, I.; Davidek, T.; Devaud, S.; Sagalowicz, L.; Leser, M. E.; Michel, M. Formation of 4-Hydroxy-5-Methyl-3(2H)-Furanone (Norfuraneol) in Structured Fluids. Dev. Food Sci. 2006, 43, 347-350.

(29) Mottram, D. S. The Maillard Reaction: Source of Flavour in Thermally Processed Foods. Flavours and Fragrances: Chemistry, Bioprocessing and Sustainability; Springer: Berlin, Heidelberg, 2007; pp 269-283.

(30) Hofmann, T.; Schieberle, P. Formation of Aroma-Active Strecker-Aldehydes by a Direct Oxidative Degradation of Amadori Compounds. J. Agric. Food Chem. 2000, 48, 4301-4305.

(31) Pattison, D. I.; Rahmanto, A. S.; Davies, M. J. Photo-Oxidation of Proteins. Photochem. Photobiol. Sci. 2012, 11, 38-53.

(32) Shi, Y.; Liang, R.; Chen, L.; Liu, H.; Goff, H. D.; Ma, J.; Zhong, F. The Antioxidant Mechanism of Maillard Reaction Products in Oilin-Water Emulsion System. Food Hydrocolloids 2019, 87, 582-592.

(33) Dong, S.; Panya, A.; Zeng, M.; Chen, B.; McClements, D. J.; Decker, E. A. Characteristics and Antioxidant Activity of Hydrolyzed $\beta$-Lactoglobulin-Glucose Maillard Reaction Products. Food Res. Int. 2012, 46, 55-61.
(34) Baglioni, P.; Dei, L.; Rivara-Minten, E.; Kevan', L. Mixed Micelles of SDS/C12E6 and DTAC/C12E6 Surfactants. J. Am. Chem. Soc. 1993, 115, 4286.

(35) Porter, W. L.; Black, E. D.; Drolet, A. M. Use of Polyamide Oxidative Fluorescence Test on Lipid Emulsions: Contrast in Relative Effectiveness of Antioxidants in Bulk Versus Dispersed Systems. J. Agric. Food Chem. 1989, 37, 615-624.

(36) Yaghmur, A.; Aserin, A.; Garti, N. Furfural-Cysteine Model Reaction in Food Grade Nonionic Oil/Water Microemulsions for Selective Flavor Formation. J. Agric. Food Chem. 2002, 50, 28782883.

(37) Kiralan, S. S.; Doğu-Baykut, E.; Kittipongpittaya, K.; McClements, D. J.; Decker, E. A. Increased Antioxidant Efficacy of Tocopherols by Surfactant Solubilization in Oil-in-Water Emulsions. J. Agric. Food Chem. 2014, 62, 10561-10566.

(38) Kranz, M.; Hofmann, T. Food-Grade Synthesis of MaillardType Taste Enhancers Using Natural Deep Eutectic Solvents (NADES). Molecules 2018, 23, 261.

(39) Bravo-Díaz, C.; Romsted, L. S.; Liu, C.; Losada-Barreiro, S.; Pastoriza-Gallego, M. J.; Gao, X.; Gu, Q.; Krishnan, G.; Sánchez-Paz, V.; Zhang, Y.; et al. To Model Chemical Reactivity in Heterogeneous Emulsions, Think Homogeneous Microemulsions. Langmuir 2015, 31, 8961-8979.

(40) Romsted, L. S.; Bravo-Díaz, C. Modeling Chemical Reactivity in Emulsions. Curr. Opin. Colloid Interface Sci. 2013, 18, 3-14.

(41) Borrelli, R. C.; Visconti, A.; Mennella, C.; Anese, M.; Fogliano, V. Chemical Characterization and Antioxidant Properties of Coffee Melanoidins. J. Agric. Food Chem. 2002, 50, 6527-6533.

(42) Rufián-Henares, J. A.; Morales, F. J. Functional Properties of Melanoidins: In Vitro Antioxidant, Antimicrobial and Antihypertensive Activities. Food Res. Int. 2007, 40, 995-1002.

(43) Kanzler, C.; Schestkowa, H.; Haase, P. T.; Kroh, L. W. Formation of Reactive Intermediates, Color, and Antioxidant Activity in the Maillard Reaction of Maltose in Comparison to d -Glucose. J. Agric. Food Chem. 2017, 65, 8957-8965.

(44) Osada, Y.; Shibamoto, T. Antioxidative Activity of Volatile Extracts from Maillard Model Systems. Food Chem. 2006, 98, 522528

(45) Chen, X.-M.; Kitts, D. D. Antioxidant Activity and Chemical Properties of Crude and Fractionated Maillard Reaction Products Derived from Four Sugar-Amino Acid Maillard Reaction Model Systems. Ann. N.Y. Acad. Sci. 2008, 1126, 220-224.

(46) Yuji, H.; Weiss, J.; Villeneuve, P.; López Giraldo, L. J.; Figueroa-Espinoza, M.-C.; Decker, E. A. Ability of Surface-Active Antioxidants to Inhibit Lipid Oxidation in Oil-in-Water Emulsion. J. Agric. Food Chem. 2007, 55, 11052-11056.

(47) Laguerre, M.; Tenon, M.; Bily, A.; Birtić, S. Toward a Spatiotemporal Model of Oxidation in Lipid Dispersions: A Hypothesis-Driven Review. Eur. J. Lipid Sci. Technol. 2020, 122, 1900209. 